\title{
Hybrid heuristic algorithm for multi-objective scheduling problem
}

\author{
PENG Jian'gang*, LIU Mingzhou, ZHANG Xi, and LING Lin
}

School of Mechanical Engineering, Hefei University of Technology, Hefei 230009, China

\begin{abstract}
This research provides academic and practical contributions. From a theoretical standpoint, a hybrid harmony search (HS) algorithm, namely the oppositional global-based HS (OGHS), is proposed for solving the multi-objective flexible job-shop scheduling problems (MOFJSPs) to minimize makespan, total machine workload and critical machine workload. An initialization program embedded in opposition-based learning (OBL) is developed for enabling the individuals to scatter in a well-distributed manner in the initial harmony memory (HM). In addition, the recursive halving technique based on opposite number is employed for shrinking the neighbourhood space in the searching phase of the OGHS. From a practice-related standpoint, a type of dual vector code technique is introduced for allowing the OGHS algorithm to adapt the discrete nature of the MOFJSP. Two practical techniques, namely Pareto optimality and technique for order preference by similarity to an ideal solution (TOPSIS), are implemented for solving the MOFJSP. Furthermore, the algorithm performance is tested by using different strategies, including $\mathrm{OBL}$ and recursive halving, and the OGHS is compared with existing algorithms in the latest studies. Experimental results on representative examples validate the performance of the proposed algorithm for solving the MOFJSP.
\end{abstract}

Keywords: flexible job-shop scheduling, harmony search (HS) algorithm, Pareto optimality, opposition-based learning.

DOI: $10.21629 / J S E E .2019 .02 .12$

\section{Introduction}

The traditional job-shop scheduling problem (JSP) is a classic discrete combinatorial optimization problem and has been attracting growing interest. To cater to the complexity of the real world, manufacturing systems need to be increasingly flexible [ $1-5]$. The flexible JSP (FJSP) comes naturally, where operations are allowed to be processed on any machine among an available machine set rather than on a specified one. Compared with the traditional JSP,

\footnotetext{
Manuscript received May 08, 2017.

*Corresponding author.

This work was supported by the National Key Research and Development Program of China (2016YFD0700605), the Fundamental Research Funds for the Central Universities (JZ2016HGBZ1035), and the Anhui University Natural Science Research Project (KJ2017A891).
}

which is a well-known non-deterministic polynomical (NP)-hard [6], the FJSP resembles real-world production scheduling more because of the assignment of the appropriate machine for each operation; the FJSP is therefore essentially NP-hard too.

A polynomial algorithm was initially proposed by Brucker et al. for solving the FJSP with two jobs [7]. Subsequently, meta-heuristic methods grew in popularity and usage for solving the FJSP and were widely utilized in finding reasonably good schedules with acceptable computational expense instead of determining exact solutions. Solving the FJSP should generally fulfill the operation arrangement and the machine assignment. The methodologies of solving the FJSP can be classified into two main categories, namely hierarchical and integrated methods, according to the principle of simultaneously considering the operation arrangement and the machine assignment sub-problems. Specifically, the former category treats the two sub-problems separately, and the latter, simultaneously. Hierarchical methods were originally introduced by Brandimarte [8]; they decomposed the FJSP into two subproblems to mitigate the solving complexity. The operation arrangement sub-problem is solved by using some dispatching rules, and the machine assignment sub-problem is solved by using the tabu search (TS) algorithm. Many researchers have concentrated on solving the FJSP by employing integrated methods. Seyed et al. implemented the biogeography-based optimisation algorithm [9]. Ishikawa et al. devised a multi-space competitive distributed genetic algorithm [10]. Harmony search (HS), particle swarm optimization (PSO) and greedy algorithm were considered by Yuan et al. [11], Singh et al. [12] and Mati et al. [13], respectively. Research results demonstrate that integrated methods perform better than hierarchical methods do.

Real-world scheduling problems are multi-objective by nature [14-17]. Each particular decision-maker aims to maximize his or her own interest. However, decisionmakers in scheduling problems substantially differ. Given 
that some conflicted objectives frequently need to be optimized simultaneously in practical situations, the multiobjective FJSP (MOFJSP) has become the latest highlighted issue. The MOFJSP, which contains the optimization and decision-making of schemes, is a complicated FJSP. The complete procedure of solving the MOFJSP can be generally divided into two areas, namely solving multiobjective optimization scheduling schemes and determining the most appropriate scheduling scheme among the optimal solutions.

Multi-objective optimisation problems (MOPs) are generally solved with the weighted aggregation or Pareto optimality. In recent years, Pareto optimality has been applied in the field of MOPs and generated many classic algorithms. Zitzler et al. [18], Corne et al. [19], Srinivas et al. [20] and Deb et al. [21] proposed Pareto-based evolutionary algorithms, such as the strength Pareto evolutionary algorithm (SPEA), the Pareto envelope-based selection algorithm (PESA)-II, the non-dominated sorting genetic algorithm (NSGA) and NSGA-II, respectively. NSGA-II is currently considered by many researchers worldwide due to its superior performance.

Many meta-heuristic approaches have been adopted for solving MOPs in view of their excellent performance in solving single-objective optimization problems. Among these, evolutionary algorithms seem particularly suitable for solving MOPs because they deal simultaneously with a set of trade-off solutions in a certain population. Li et al. [22], Rahmati et al. [23], Wang et al. [24] and Rohaninejad et al. [25] designed hybrid algorithms based on the genetic algorithm to successfully solve the MOFJSP. Furthermore, some meta-heuristics based on local search methods, such as TS and simulated annealing (SA), have been applied recently for the FJSP. Jia et al. [26], Zhang et al. [27], Vilcot et al. [28] and Li et al. [29] used TS in solving the MOFJSP. Kaplanoglu [30] presented an object- oriented approach for the MOFJSP along with SA. Xia et al. [31] implemented SA to enhance the local search performances of PSO, and Low et al. [32] designed a hybrid SA-TS algorithm to solve the MOFJSP. Gao et al. [33] investigated a Pareto-based HS algorithm to solve the MOFJSP.

In practice, the MOFJSP is a sophisticated optimization problem which involves not only MOPs but also multiattribute decision-making (MADM). Firstly, the performance of the algorithm, keeping a balance between the global exploration and local exploitation in the universe of discourse, is significant to meta-heuristics [34]. Secondly, the convergence of the algorithm and the diversity of nondominated solutions become essential problems because the Pareto optimality approach generates a large number of non-dominated solutions in MOPs. Finally, the selec- tion of the most appropriate scheduling scheme according to decision-makers' intention is indispensable.

In this research, an oppositional global-based HS (OGHS) algorithm integrating HS and opposition-based learning (OBL) is proposed for solving the MOFJSP for three common objective functions. The HS algorithm, which is simple, easily implemented, and an excellent meta-heuristic based on population, has been successfully applied for optimizing real-world problems [11]. In the OGHS, significant initialization program-embedded OBL is developed for obtaining a satisfactory diversity of individuals in a population. Recursive halving based on OBL is implemented for shrinking the neighbourhood space rapidly. The proposed algorithm not only guarantees the diversity of non-dominated solutions but also ensures rapid convergence. The technique for order preference by similarity to an ideal solution (TOPSIS) is adopted for seeking the most appropriate scheduling scheme for the decisionmakers of the MOFJSP.

The remainder of this paper is organized as follows. In Section 2, the mathematical model of the MOFJSP is described, and some basic concepts are defined. Section 3 introduces the groundwork related to the proposed algorithm. In Section 4, the particular scheme of the OGHS algorithm for the MOFJSP is elaborated. The performance examination of the OGHS and the comparison of its results with those of other approaches on certain benchmarks are presented in Section 5. Section 6 explains the decision-making process of the most appropriate scheduling scheme. Conclusions are summarized, and some future investigation perspectives are described in Section 7.

\section{Problem description}

The FJSP of $n$ jobs on $m$ machines is to organize execution as follows. Each job, denoted by $J_{i}(1 \leqslant i \leqslant n)$, has a pre-defined sequence $n_{i}$ of non-preemptible ordered operations. The machine is noted $M_{k}, k \in\{1,2, \ldots, m\}$. The execution of each operation of job $J_{i}$ (noted $O_{i j}$ ) is permitted to be processed by one machine in a set of available machines (called $M_{i j}$ ), and $M_{i j} \subseteq M_{k}$ obviously. The FJSP naturally aims to allocate the operations for each job to available equipments, to determine their starting and completion time and to further optimize the pre-determined objectives.

The FJSP is classified into the total FJSP and the partial FJSP [35]. In the total FJSP, each operation is manipulated by any machine, whereas in the partial FJSP, at least an operation cannot be processed on all machines. At any rate, processes and machine constraints must be satisfied in a real environment.

In this work, our model of the MOFJSP, which involves 
three sub-objectives, is provided as follows:

$$
\left\{\begin{array}{l}
\left.f_{1}=\min C_{\max }=\min (\max )\left\{c_{i} \mid i=1,2, \ldots, n\right\}\right) \\
f_{2}=\min W_{T}=\min \left(\sum_{i=1}^{n} \sum_{j=1}^{n_{i}} \sum_{s=1}^{m} p_{i j s} x_{i j s}\right) \\
f_{3}=\min W_{M}=\min \left(\max \left\{\sum_{i=1}^{n} \sum_{j=1}^{n_{i}} p_{i j s} x_{i j s}\right\}\right)
\end{array}\right.
$$

where $C_{\max }$ is the maximum makespan, $W_{T}$ is the total workload of machines, $W_{M}$ is the workload of the critical machine, $C_{i}$ is the makespan of $J_{i}, n_{i}$ denotes the total number of job $J_{i}, m$ represents the total number of machines, $p_{i j s}$ denotes the processing time of $O_{i j}$ on machine $s$ and $x_{i j s}$ is a decision variable.

A partial FJSP example with three jobs and three machines is listed in Table 1. The symbol "-" indicates that the assignment is impossible in this example.

Table 1 Execution time of an example of $3 \times 3$ partial FJSP

\begin{tabular}{ccccc}
\hline Job & Operation & $M_{1}$ & $M_{2}$ & $M_{3}$ \\
\hline \multirow{3}{*}{$J_{1}$} & $O_{11}$ & 3 & - & 2 \\
& $O_{12}$ & 5 & 2 & 3 \\
& $O_{13}$ & - & 2 & 4 \\
\hline \multirow{2}{*}{$J_{2}$} & $O_{21}$ & 4 & 5 & 3 \\
& $O_{22}$ & 7 & - & 9 \\
\hline \multirow{3}{*}{$J_{3}$} & $O_{31}$ & 4 & 2 & 1 \\
& $O_{32}$ & - & 5 & 3 \\
& $O_{33}$ & 4 & - & 5 \\
\hline
\end{tabular}

\section{Related work}

In the proposed algorithm, HS is considered the main algorithm. Pareto optimality is used to achieve the convergence of the algorithm through guiding the evolution of the population towards the Pareto front. OBL improves the quality of distribution by enhancing the diversity of the nondominated solutions. Furthermore, recursive halving based on OBL accelerates the convergence speed by shrinking the neighbourhood space.

\subsection{Pareto optimality}

The idea of Pareto optimality is originally proposed by Edgeworth, generalized later by Vilfredo Pareto and eventually became a widely accepted concept, namely Pareto optimality [36-38].

A minimized MOP can be generally described as follows:

$$
\text { Minimize } y=f(\boldsymbol{X})=\left(f_{1}(\boldsymbol{x}), f_{2}(\boldsymbol{x}), \ldots, f_{L}(\boldsymbol{x})\right)
$$

where $\boldsymbol{x} \in \Re^{p}$ is a possible solution in the universe of discourse, $\Re$ is the solution space and $p$ represents the quantity of multi-dimensional $\boldsymbol{x}$. Moreover, $y \in \Re^{L}$, where
$L$ denotes the quantity of sub-objectives. $f_{q}(\boldsymbol{x})$ is the $q$ th sub-objective function, for $1 \leqslant q \leqslant L$. In practice, the feasible solutions are increased exponentially, whereas multiple sub-objectives are optimized simultaneously. Generally, a set of trade-off solutions can be obtained according to the conflicted sub-objectives rather than an exact solution. The trade-off solutions, also called Pareto optimal solutions [39], are at least better in one sub-objective than the others in the universe of discourse. Therefore, the Pareto optimal solutions in MOPs are those that cannot be improved along any dimension without simultaneously being deteriorated along other dimensions. The trade-off solutions are also defined as non-dominated solutions. Additionally, some Pareto optimality concepts used in this paper can be formulated as follows.

Definition 1 Pareto dominance. In a minimized MOP, a feasible solution $x_{A}$ is defined to dominate another feasible solution $x_{B}$, denoted by

$$
x_{A} \prec x_{B}
$$

satisfying $\forall i=1,2, \ldots, m, \quad f_{i}\left(x_{A}\right) \leqslant f_{i}\left(x_{B}\right) \wedge \exists j=$ $1,2, \ldots, m, f_{j}\left(x_{A}\right) \leqslant f_{j}\left(x_{B}\right)$.

Definition 2 Non-dominated solution. A feasible solution $x^{*}$ is deemed as a non-dominated solution if and only if no feasible solution $x^{\prime}$ which dominates $x^{*}$ in a minimized MOP, i.e.,

$$
\neg \exists x^{\prime}, \quad x^{\prime} \prec x^{*} .
$$

Definition 3 Non-dominated solution set (NDSS). The NDSS $P^{*}$ in a minimized MOP is defined as

$$
P^{*} \stackrel{\text { def }}{=}\left\{\boldsymbol{x} \in \Re^{p} \mid \neg \exists x^{\prime} \in \Re^{p}, f\left(x^{\prime}\right) \leqslant f(\boldsymbol{x})\right\} .
$$

\subsection{HS algorithm}

\subsubsection{Standard HS algorithm}

The HS algorithm was first inspired by Geem et al. [40]. Considered as one of the nearest meta-heuristics based on population evolvement methods due to its simplicity and easiness of implementation, the HS algorithm has drawn growing interest and extensive application successfully. Its effectiveness and advantage have been demonstrated in various practical applications, such as function optimization, FJSP [11] and engineering optimization [41]. To achieve a perfect state of music, musicians improvise their instruments' pitches. On the basis of the improvisation process done by skilled musicians, Geem [40] formalized three corresponding components in HS, specifically the generation of harmony memory (HM), the pitch adjustment and the replacement of HM. The first component guarantees that the best harmony will be preserved in a new HM. A new solution is produced via varying the 
pitches slightly by a small random amount in the second component, where the randomization selection is allowed to increase the diversity of the solutions for the global optimality. The worst existing harmony is substituted by a new candidate harmony in the last component.

\subsubsection{Variant of HS}

To alleviate the problem of parameter adjustment which was difficult to specify a priori in HS, Mahdavi et al. [42] investigated a variant of HS, which updated dynamically the pitch adjusting rate (PAR) and the distance bandwidth. Mahamed et al. [43] designed a global-based HS (GHS) algorithm wherein the pitch adjustment was modified and the best harmony was straightforwardly reserved in the HM. Importantly, the GHS works efficiently not only in the continuous universe but also in the discrete space.

\subsection{OBL}

\subsubsection{Definition of OBL}

Tizhoosh [44] proposed OBL, which has been used to improve evolutionary algorithms such as PSO [45,46], differential evolution [47] and biogeography-based optimization $[48,49]$. The basic idea of OBL is to use opposite numbers instead of random ones to quickly evolve the population in the evolutionary process. For convenience of description, the multi-dimensional opposite number is defined as follows.

Definition 4 In an $n$-dimensional space, $P\left(x_{1}, x_{2}, \ldots\right.$, $\left.x_{n}\right)$ is a certain point, $x_{1}, \ldots, x_{n} \in \Re^{n}$ and $x_{i} \in\left[a_{i}, b_{i}\right]$. Its opposite point $\widehat{P}$ is defined by coordinates $\widehat{x}_{1}, \ldots, \widehat{x}_{n}$ as

$$
\widehat{x}_{i}=a_{i}+b_{i}-x_{i}, \quad i=1,2, \ldots, n .
$$

A certain point $x \in[a, b]$ and $c=(a+b) / 2$ in a onedimensional space, and its opposite point, $\widehat{x}$, is illustrated in Fig. 1.

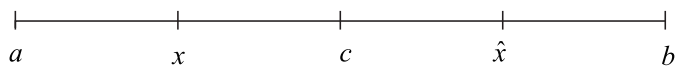

Fig. 1 Opposite point

\subsubsection{Recursive halving based on OBL}

In the search space, a theoretical optimal solution must be considered, such as $x_{\mathrm{opt}}$, which is obtained by finite iterations. However, searching for $x_{\mathrm{opt}}$ is a time-consuming process because of exponentially large non-dominated solutions in an MOP. In the MOFJSP, the trade-off solutions exist in NDSS as alternative scheduling schemes. Any nondominated solution comes into being if and only if at least one of its objective is demoted while some others improved. Therefore, the non-dominated solutions are worthy of solution rather than $x_{\mathrm{opt}}$ in view of the computational cost in engineering practice. Point $x$ is an initial number generated by random selection, whereas its opposite number $\widehat{x}$ is produced following Definition 4. $x$ or $\widehat{x}$ does not dominate each other in the iteration process. Meanwhile, the search range can be recursively halved by the nondominated solutions [44], as illustrated in Fig. 2. See the specific steps of Algorithm 2. The optimal solution $x_{\mathrm{opt}}$, which is theoretically approached by $x$ or $\widehat{x}$ in the searching process, only illustrates the existence of the optimal solution in Fig. 2.

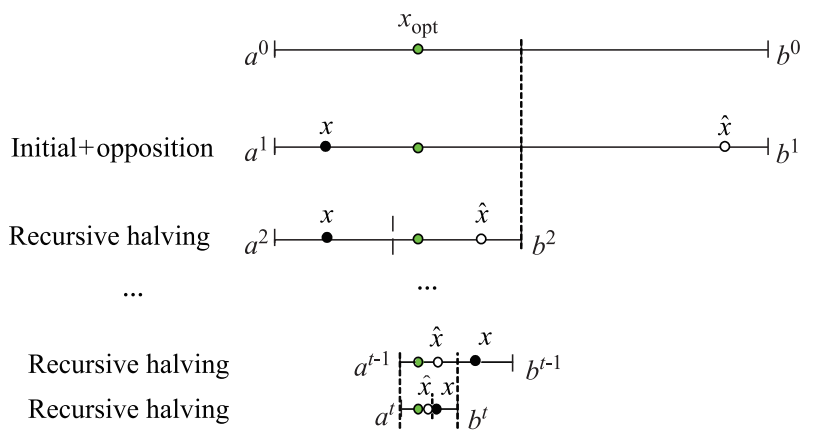

Fig. 2 Schematic diagram of recursive halving based on OBL

\section{OGHS approach}

This section presents an exhaustive explanation of the OGHS algorithm. The MOFJSP is a complex combinatorial optimization problem. A discrete HS, namely GHS [43], is utilized in the OGHS. OBL is exploited to improve the quality of the initial population distribution and to reduce the search range by a recursive halving of the search space. The specific OGHS algorithm is elaborated as follows.

\subsection{OGHS algorithm framework}

The OGHS algorithm framework is illustrated in Fig. 3, which is integrated with both GHS and OBL.

\subsection{Harmony vector representation for MOFJSP}

A harmony vector

$$
\boldsymbol{X}=\{x(1), x(2), \ldots, x(s), x(s+1), \ldots, x(2 s)\}
$$

where $-\zeta \leqslant x(j) \leqslant \zeta, j=1,2, \ldots, 2 s$. To solve the MOFJSP, $\boldsymbol{X}$ is firstly translated into a type of dual vector code [11] and subsequently decoded to a feasible scheme. 


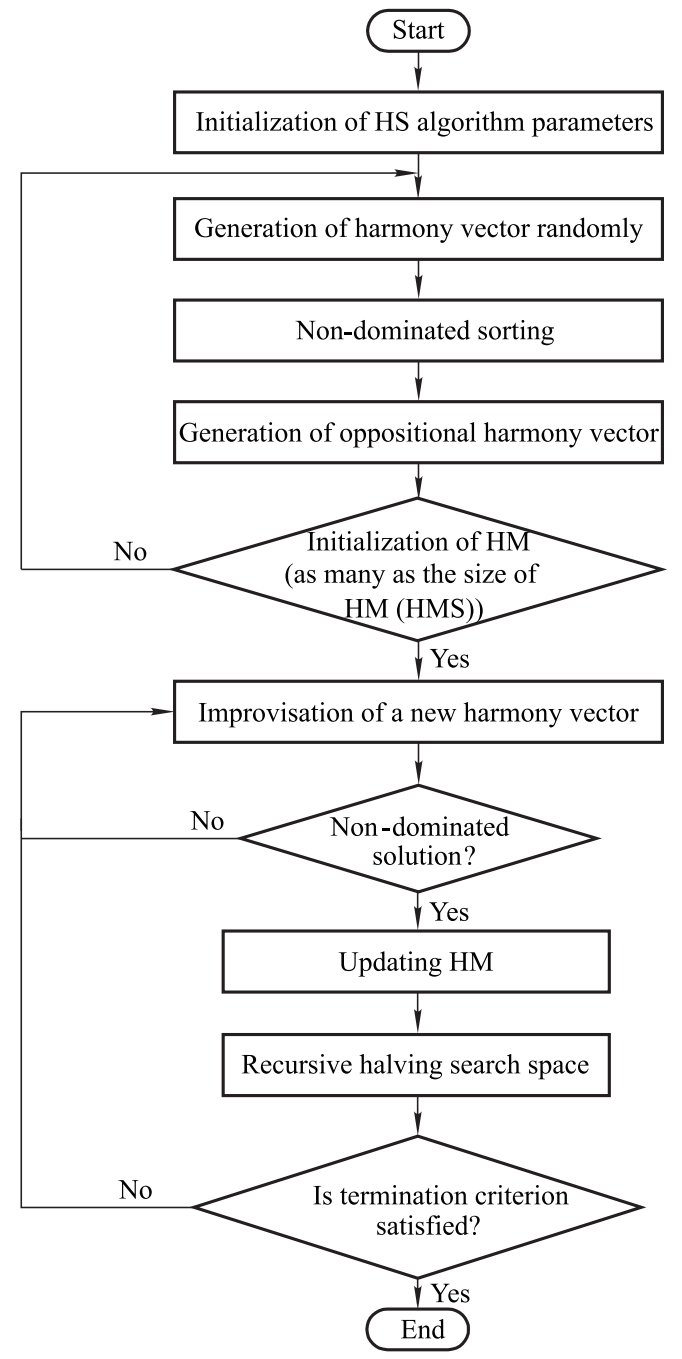

Fig. 3 Flow chart of OGHS

\subsubsection{Encoding procedure}

The harmony vector representation of the MOFJSP consists of a dual vector code [11], including the operation sequence and machine allocation corresponding to the operation arrangement sub-problem and the machine assignment sub-problem. The vector $\boldsymbol{X}$ is $2 s$-dimensional, where $s$ is the total number of all operations in the MOFJSP. The first half part of $\boldsymbol{X}$

$$
\boldsymbol{X}^{(1)}=\{x(1), x(2), \ldots, x(s)\}
$$

describes the operation sequence information, whereas the second part of $\boldsymbol{X}$

$$
\boldsymbol{X}^{(2)}=\{x(s+1), x(s+2), \ldots, x(2 s)\}
$$

represents the machine allocation information. For making encoding much more accessible to interpret, each operation is marked with a definite ID according to the job position, and evidently, the total number of the definite ID is equal to the quantity of all the operations. Therefore, each operation can be demonstrated by one definite ID. For instance, the operation position 7 , which corresponds to the definite ID $=7$, signifies the second operation of the third job, i.e., $\mathrm{O}_{32}$, as shown in Fig. 4. The operation sequence vector

$$
\boldsymbol{V}_{o}=\{v(1), v(2), \ldots, v(s)\}
$$

can be translated into an operation sequence [50], i.e., [1, $1,1,2,2,3,3,3]$, where all operations are marked with the same number satisfying the precedence constraints. Consequently, operation sequences ensure the feasibility of the schedule scheme without doubt.

$$
\begin{aligned}
& \begin{array}{lllllllll}
\text { Operation position } & 1 & 2 & 3 & 4 & 5 & 6 & 7 & 8
\end{array} \\
& \begin{array}{lllllllll}
\text { Definite ID } & 1 & 2 & 3 & 4 & 5 & 6 & 7 & 8
\end{array} \\
& \text { Job demonstration } \quad \begin{array}{lllllllll}
J_{1} & J_{1} & J_{1} & J_{2} & J_{2} & J_{3} & J_{3} & J_{3}
\end{array} \\
& \text { Operation sequence } \quad \begin{array}{|l|l|l|l|l|l|l|l|}
\hline 1 & 1 & 1 & 2 & 2 & 3 & 3 & 3 \\
\hline
\end{array} \\
& \text { Operation demonstration } O_{11} O_{12} O_{13} O_{21} O_{22} O_{31} O_{32} O_{33} \\
& \text { Machine allocation } \quad \begin{array}{|l|l|l|l|l|l|l|l|}
2 & 3 & 1 & 3 & 1 & 2 & 2 & 1 \\
\hline
\end{array} \\
& \text { Alternative machine set }\left|M_{1 !} M_{3}\right| M_{1}\left\lceil M_{2 !} M_{-}\right\rfloor
\end{aligned}
$$

Fig. 4 Illustration of dual vector code

For the machine allocation vector,

$$
\boldsymbol{V}_{M}=\{u(1), u(2), \ldots, u(s)\}
$$

is an arrangement of $s$ integer values, i.e., a specific permutation $[2,3,1,3,1,2,2,1]$, as shown in Fig. 4. The machine allocation vector, $u(j)(1 \leqslant j \leqslant s)$, indicates that the $u(j)$ th machine is chosen by the operation $j$. For instance, $u(2)=3$ reveals that machine $M_{3}$ is chosen for the operation $O_{12}$, as shown in Fig. 4.

\subsubsection{Mapping the harmony vector into dual vector}

The random keys [51] based on the largest position value rule are applied to implement the mapping procedure of the operation sequence. Firstly, a random number drawn uniformly from $[-1,1]$ is assigned to each ID. Secondly, the position is visited in a non-increasing value. Thirdly, the operation ID is converted by using the job symbol. Finally, the feasible operation permutation is generated according to the constraints of the same job.

For instance, the conversion of the operation permutation for the problem in Table 1 is illustrated in Fig. 5. In detail, a random number is assigned to each ID as follows:

$$
\boldsymbol{X}^{(1)}=\{0.5,0.4,0.1,-0.1,0.6,0.7,-0.3,-0.4\} .
$$




\begin{tabular}{|c|c|c|c|c|c|c|c|c|}
\hline Operation position & 1 & 2 & 3 & 4 & 5 & 6 & 7 & 8 \\
\hline $\boldsymbol{X}^{(1)}$ & 0.5 & 0.4 & 0.1 & -0.1 & 0.6 & 0.7 & -0.3 & -0.4 \\
\hline Sorting & 0.7 & 0.6 & 0.5 & 0.4 & 0.1 & -0.1 & -0.3 & -0.4 \\
\hline Operation ID arrangement & 6 & 5 & 1 & 2 & 3 & 4 & 7 & 8 \\
\hline Operation arrangement & $O_{31}$ & $O_{22}$ & $O_{11}$ & $O_{12}$ & $O_{13}$ & $O_{21}$ & ${ }_{1} O_{32}$ & $O_{33}$ \\
\hline Operation sequence & 3 & 2 & 1 & 1 & 1 & 2 & 3 & 3 \\
\hline
\end{tabular}

Fig. 5 Generation procedure of feasible operation arrangement

All random numbers are sorted in the descending order as $[0.7,0.6,0.5,0.4,0.1,-0.1,-0.3,-0.4]$.

Hence, the corresponding ID arrangement and the operation arrangement are generated respectively as $[6,5,1,2$, $3,4,7,8]$ and $\left[O_{31}, O_{22}, O_{11}, O_{12}, O_{13}, O_{21}, O_{32}, O_{33}\right]$.

All the operations existing in a certain job are marked with the same numerical code, and subsequently, the operation sequence is acquired as [3, 2, 1, 1, 1, 2, 3, 3].

Consequently, the feasible operation arrangement is generated, i.e., $\left[\mathrm{O}_{31}, \mathrm{O}_{21}, \mathrm{O}_{11}, \mathrm{O}_{12}, \mathrm{O}_{13}, \mathrm{O}_{22}, \mathrm{O}_{32}, \mathrm{O}_{33}\right]$.

The mapping procedure of machine allocation is implemented as follows: converting the real number $x(s+j) \in$ $[-\zeta, \zeta]$ to the integer $u(j) \in[1, e(j)]$ by linear transformation, where $e(j)$ denotes the largest number of the machine in its feasible machine set for the operation $j$. The values of $u(j)$ are computed by Yuan et al. [11].

\subsubsection{Decoding procedure}

The plug-greedy decoding algorithm is introduced to ensure that the decoded solutions are active schedules. The basic procedure is described as follows: the decoding sequence abides by the arrangement order of operations. The first operation in the arrangement is arranged. Then, the second one in the arrangement is planned on the feasible machine in the earliest competent time, and so on. For every machine, the earliest available idle time interval is arranged preferentially. Without such an idle time at present, the operation is executed at the end of the machine.

\subsection{Initialization of HM}

Population initialization is of great importance in the HS algorithm because the quality of the initial solutions in HM considerably affects the convergence rate and solution distribution. Random initialization has been generally adopted in the absence of any priori information. Unfortunately, this method cannot effectively guarantee that the initial population obtains the information of the solution space, and the algorithm efficiency would be consequently limited to a certain extent. The initialization strategy integrating random and OBL technique is applied to allow the initial population to use the information of the search space. Furthermore, the oppositional vector is produced according to the initial feasible solution. And the solutions in $\mathrm{HM}$ are sorted according to Definition 1.

To guarantee the feasibility of the solutions in the OGHS, the dual vector code of the initial solution must satisfy the rule that the number of operation of job $J_{i}$ is equal to $n_{i}$ and the total number of all the operations is equal to $s$.

\subsubsection{Generating a random code}

A random harmony vector

$$
\boldsymbol{X}=\{x(1), x(2), \ldots, x(n)\}
$$

can be produced by using the following equation:

$$
\begin{gathered}
x(j)=x_{\min }(j)+\left(x_{\max }(j)-x_{\min }(j)\right) \times \operatorname{rand}(0,1), \\
j=1,2, \ldots, n
\end{gathered}
$$

where $x_{\min }$ and $x_{\max }$ represent the bound values for the positions and rand $(0,1)$ denotes a random function valued from 0 to 1 .

The feasibility of the random solution is verified through traversing all the operations in order in the dual vector code. In case the number of operations of job $J_{i}$ is not equal to $n_{i}$ or the total number of all the operations is not equal to $s$, the code is adjusted according to the initial number of $n_{i}$ or of $s$ correspondingly.

\subsubsection{Oppositional operation sequence}

Consciously, the non-dominated solution has an important effect on the global optimization. Then, OBL is employed to generate an oppositional harmony vector according to the non-dominated one. Because the oppositional harmony vector in our algorithm is only involved with the operation sequence, the operations are traversed in order and sorted with accelerating position values. For example, the oppositional operation arrangement in Fig. 5 is easily received as $[3,3,2,1,1,1,2,3]$. The procedure generating and oppositional operation sequence is shown in Algorithm 1.

Algorithm 1 The procedure generating oppositional operation permutation

Step 1 Generate $\boldsymbol{X}^{(1)}=-2 \operatorname{rand}(1, N)+1$;

Step $2[A, B]=\operatorname{Sort}\left(\boldsymbol{X}^{(1)}\right)$;

Step 3 Generate operation arrangement according to $B$;

Step 4 Transform operation arrangement into the operation sequence;

Step 5 Generate oppositional operation arrangement according to the operation sequence.

\subsubsection{Generating harmony vector}

The procedure generating harmony vector representation includes two parts, namely the operation sequence and ma- 
chine assignment. The transformation is derived from the approach of Yuan et al. [11].

\subsection{Improvisation of a new harmony}

Similar to the FJSP, the MOFJSP is a classic discrete combinatorial optimisation problem in the universe of discourse. As mentioned in 3.2.2, GHS can efficiently work in the discrete search space. Memory consideration, pitch adjustment and random selection constitute the main procedure of improvising a new harmony in GHS.

Considering the memory, a new vector $\boldsymbol{X}^{\prime}=$ $\left(x^{\prime}(1), x^{\prime}(2), \ldots, x^{\prime}(2 s)\right)$ is generated from HM. The decision variable $x^{\prime}(i)$ is determined through any value in the range $\left(x^{\prime}(i)-x^{\mathrm{HMS}}(i)\right)$. The HMS parameter indicates the size of HM. The probabilities of selecting, either the historic HM or the entire search space, are HM considering rate (HMCR) or 1-HMCR, respectively. The feasibility of the new vectors selected randomly from the entire search space must necessarily be verified. Specifically, the new vector representation is firstly transformed into a dual vector code and subsequently checked and adjusted according to the rule in Section 4.3.1.

After choosing a value from the HM, the new vector representation, $\boldsymbol{X}^{\prime}=\left(x^{\prime}(1), x^{\prime}(2), \ldots, x^{\prime}(2 s)\right)$, should be examined to determine whether it should be pitch-adjusted. Specifically, the probability of selecting a non-dominated solution is PAR $\times$ HMCR. Moreover, the probability of doing nothing is 1-PAR.

\subsection{Recursive halving of search space}

The effective neighbourhood of the local search is based on the recursive halving method, which is motivated by the narrowing of the search area. The procedure of generating an acceptable neighbourhood depending upon recursive halving based on OBL is shown in Algorithm 2.

Algorithm 2 The procedure of generating an acceptable neighbourhood in virtue of recursive halving based on OBL.

Step 1 Initialization:

$A_{\text {_left }}=a_{0}, B_{\text {_right }}=b_{0}, x_{\text {_random }}$.

Step 2 Definition of the opposition point of $x_{\text {_random: }}$ : $\widehat{x}_{\text {_random }}=A_{\text {_left }}+B_{\text {_right }}-x_{\text {_random}}$.

Step 3 Recursive halving based on OBL.

Update $A_{\text {_left }}$ and $B_{\text {_right }}$ by this way:

If $x_{\text {_random }}$ is non-dominated, $A_{\text {-left }}=A_{\text {-left }}$, $B_{\text {_right }}=\left(A_{\text {_left }}+B_{\text {_right }}\right) / 2$;

Else if $\widehat{x}_{\text {_random }}$ is non-dominated, $A_{-}$left $=\left(A_{\text {-left }}+\right.$ $\left.B_{\text {_right }}\right) / 2, B_{\text {_right }}=B_{\text {_right}}$.

Step 4 Update the opposition of $x_{\text {_random. }}$.

Step 5 Recursive halving until reaching the termination criterion.

\subsection{Complexities of OGHS algorithm}

The complexities of the OGHS algorithm includes the space and the time complexities.

The difference between the OGHS and the standard HS algorithm is that the former combines the OBL and the recursive halving technique, and the two strategies do not increase the storage space. Therefore, the space complexity of the OGHS and HS is barely different [52]. Because the $\mathrm{HM}$ is the population space in the iterative process, the OGHS algorithm only needs the newly generated individual space to update the original space. Therefore, the space complexity is of constant value, i.e., the space complexity of the OGHS algorithm is $O(1)$.

The time complexity of the proposed algorithm is composed of two components. One is the time complexity of the standard HS algorithm, and the other is the time complexity of non-dominated sorting. As mentioned above, the most critical operations of the HS algorithm are the generation of HM, the pitch adjustment and the replacement of HM. The HS algorithm is of one-order linear progressive time complexity of the maximum iteration number [52]. Suppose the population size is $N$ and the number of non-dominated solutions is $r$ in an iterative process of the OGHS. The preservation rate and the replacement rate of population individuals are denoted by $\alpha$ and $\beta$, respectively. Evidently, $\alpha+\beta=1$. During a non-dominated sorting phase, the second individual is compared only with the first. The third individual is compared at most with the first and the second, and so on. The $N$ th individual needs $(N-1)$ comparative operations at most. In the worst scenario, where all the individuals are non-dominated solutions, the total number of comparative times can be computed as $[1+2+3+\ldots+(N-1)]$, i.e., $N \cdot(N-1) / 2$. Thus, the time complexity of the proposed algorithm is $T(N)=N^{2}+\alpha \cdot N+\beta \cdot N$. Consequently, the time complexity of the OGHS algorithm is expressed as $O\left(r N^{2}\right)$.

\subsection{Updating of HM}

All harmony vectors are assessed after improvising a new harmony. In the current iteration, if a new harmony vector is non-dominated through the existing harmony in the $\mathrm{HM}$, the new vector is included in the HM, and the dominated vector is excluded from the HM. Otherwise, no action should be implemented.

\subsection{Termination}

If the termination criterion is satisfied, then the algorithm ends and outputs the computational results. The last HM is updated after non-dominated sorting. The archive set is the final result of the OGHS algorithm. 


\section{Experiment results and discussion}

First, this section tests the performance of the proposed algorithm by using different strategies, namely OBL and recursive halving, and subsequently analyzes the computational outcomes of the OGHS algorithm and the comparison results by using other approaches on benchmarks extracted from publications. The OGHS algorithm is manipulated in Matlab R2009a and performed on a personal computer with $3.19 \mathrm{GHz}$ CPU and 3.47 GB RAM. HMS = $50, \mathrm{HMCR}=0.95$, and $\mathrm{PAR}=0.3$.

\subsection{Performance examination for strategies}

To examine the performance of the OBL and the recursive halving technique embedded in the HS, the widely adopted multi-objective test functions of DTLZ1-4 and DTLZ7, which are suitable for evaluating population diversity and iterative convergence in a high-dimensional space [53,54], are optimized in this section. DTLZ1-4 problems are continuous functions, whereas DTLZ7 is a non-continuous function. The performance metrics of CPU time, generational distance (GD) and inverted generational distance (IGD) [55] are used to evaluate the OGHS. The five problems of DTLZ are tested by using HS, HS+OBL and HS+OBL+halving (i.e., OGHS), respectively. Each algorithm runs three times. The results, including performance metrics of CPU time and GD at the 5 000th iteration and their average values, are listed in Table 2 and Table 3 . The convergence curves, portraying the different combinations between the HS and different strategies, are illustrated in Figs. 6-10. Furthermore, the mean and variance values of IGD, corresponding to the 100th, 500th and 3 000th generations, are listed in Table 4.

Table 2 Values of CPU time at the 5 000th iteration

\begin{tabular}{lccccc}
\hline \multirow{2}{*}{ Problem } & \multirow{2}{*}{ Algorithm } & \multicolumn{4}{c}{ Time/s } \\
\cline { 3 - 6 } DTLZ1 & HS+OBL & 11.3701 & 11.5046 & 10.9282 & 11.2676 \\
& OGHS & 11.0800 & 11.1533 & 11.0643 & 11.0992 \\
\hline \multirow{3}{*}{ DTLZ2 } & HS & 11.1422 & 11.1169 & 10.9705 & 11.0765 \\
& HS+OBL & 11.0318 & 10.9662 & 11.1008 & 11.0329 \\
& OGHS & 11.1861 & 11.0559 & 11.3231 & 11.1884 \\
\hline \multirow{4}{*}{ DTLZ3 } & HS & 11.0594 & 10.8949 & 11.1800 & 11.0448 \\
& OS+OBL & 11.1275 & 11.2787 & 10.9868 & 11.1310 \\
& OGHS & 11.2756 & 10.8677 & 11.2256 & 11.1230 \\
\hline \multirow{2}{*}{ DTLZ4 } & HS & 11.2263 & 10.9678 & 10.8020 & 10.9987 \\
& OG+OBL & 11.0541 & 11.3392 & 10.8781 & 11.0905 \\
& OGH & 11.1877 & 11.3128 & 11.2001 & 11.2335 \\
\hline \multirow{2}{*}{ DTLZ7 } & HS & 11.8645 & 10.8022 & 11.9209 & 11.5292 \\
& OGHS & 10.9031 & 11.3950 & 11.0621 & 11.1201 \\
\hline
\end{tabular}

From Table 2, the values of CPU time for different combinations between the HS and different strategies at the 5 000th iteration belong to the same order of magnitude.
Table 3 Values of GD at the 5 000th iteration

\begin{tabular}{lccccc}
\hline \multirow{2}{*}{ Problem } & \multirow{2}{*}{ Algorithm } & \multicolumn{4}{c}{ GD } \\
\cline { 3 - 6 } & & 1 & 2 & 3 & Average \\
\hline \multirow{3}{*}{ DTLZ1 } & HS & 0.0183 & 0.0140 & 0.0078 & 0.0134 \\
& HS+OBL & 0.0133 & 0.0144 & 0.0078 & 0.0118 \\
& OGHS & 0.0097 & 0.0100 & 0.0089 & 0.0095 \\
\hline \multirow{3}{*}{ DTLZ2 } & HS & 0.0171 & 0.0170 & 0.0181 & 0.0174 \\
& HS+OBL & 0.0154 & 0.0149 & 0.0178 & 0.0160 \\
& OGHS & 0.0157 & 0.0164 & 0.0163 & 0.0161 \\
\hline \multirow{5}{*}{ DTLZ3 } & HS & 0.0217 & 0.0607 & 0.0811 & 0.0545 \\
& HS+OBL & 0.0186 & 0.0227 & 0.0215 & 0.0209 \\
& OGHS & 0.0193 & 0.0213 & 0.0203 & 0.0203 \\
\hline \multirow{3}{*}{ DTLZ4 } & HS & 0.1015 & 0.0511 & 0.0787 & 0.0771 \\
& HS+OBL & 0.0750 & 0.0583 & 0.0617 & 0.0650 \\
& OGHS & 0.0550 & 0.0597 & 0.0522 & 0.0556 \\
\hline & HS & 0.0434 & 0.0643 & 0.0502 & 0.0526 \\
& OG+OBL & 0.0237 & 0.0565 & 0.0523 & 0.0442 \\
& OGHS & 0.0216 & 0.0331 & 0.0356 & 0.0301 \\
\hline
\end{tabular}

The time performance of OGHS for DTLZ1 and DTLZ7 is better than the HS's though the time complexity of the OGHS is inferior to the HS's. The main reason is that recursive halving speeds up the searching process. From the values of GD at the 5 000th iteration in Table 3, the average values of the $\mathrm{HS}+\mathrm{OBL}$ algorithm are much better than those of the HS algorithm, and the average values of the OGHS algorithm outperform those of HS+OBL except DTLZ2. As shown in Figs. 6-10, the speeds of the convergence of the OGHS algorithm are considerably better than those of HS and HS+OBL. The unsatisfactory result of DTLZ4 must be interpreted because of its complicated Pareto front.

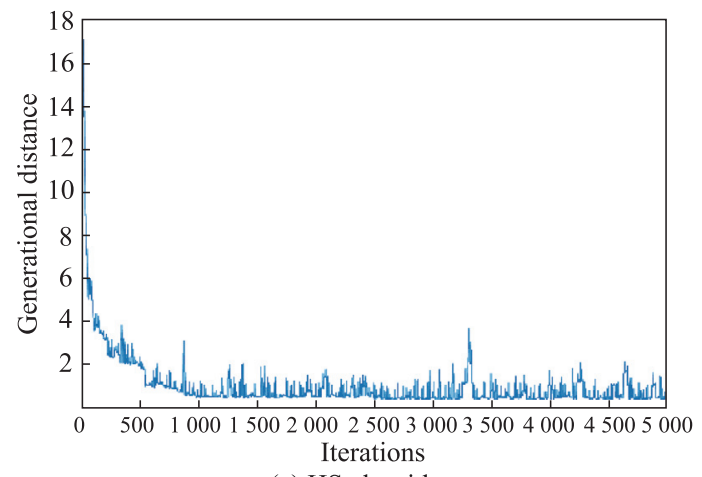

(a) HS algorithm

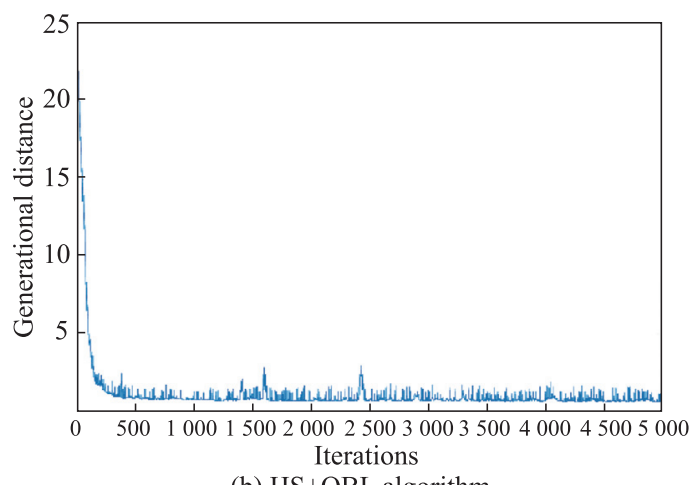

(b) HS+OBL algorithm 


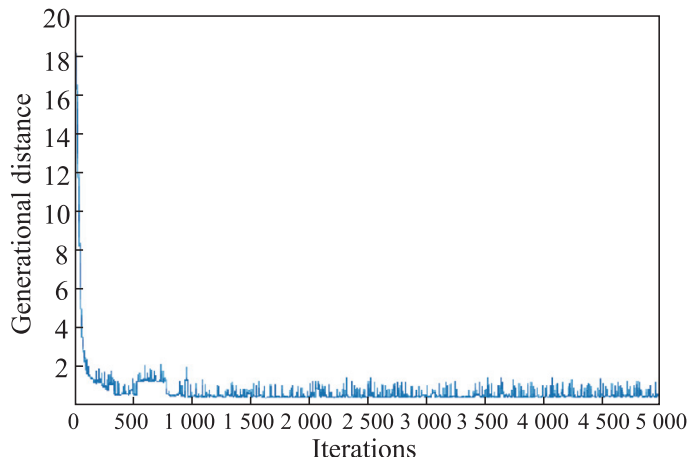

(c) $\mathrm{HS}+\mathrm{OBL}+$ halving algorithm

Fig. 6 Convergence curves of DTLZ1

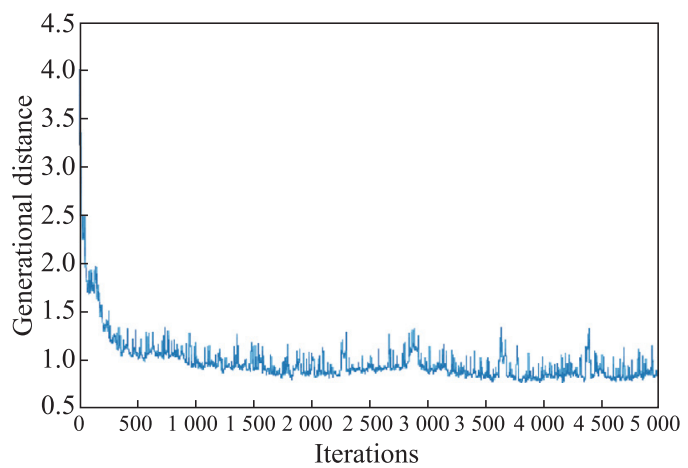

(a) HS algorithm

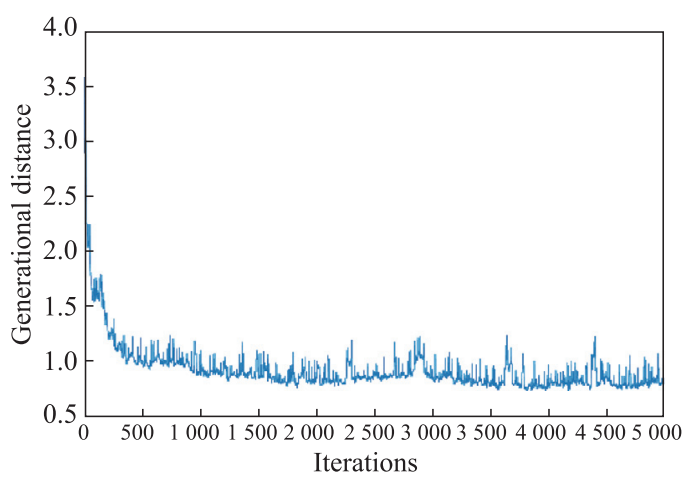

(b) HS+OBL algorithm

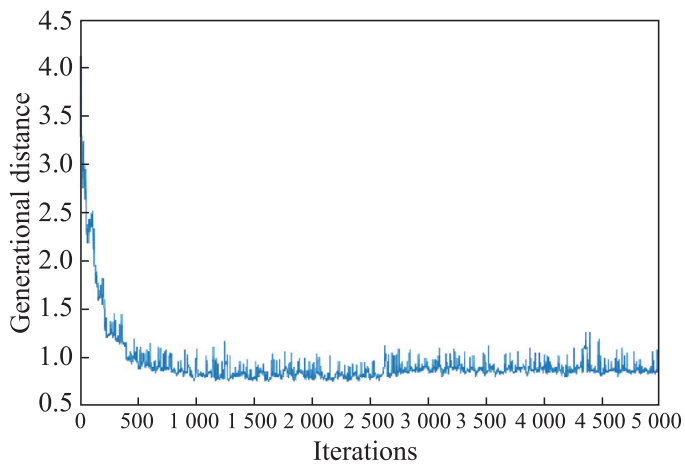

(c) $\mathrm{HS}+\mathrm{OBL}+$ halving algorithm

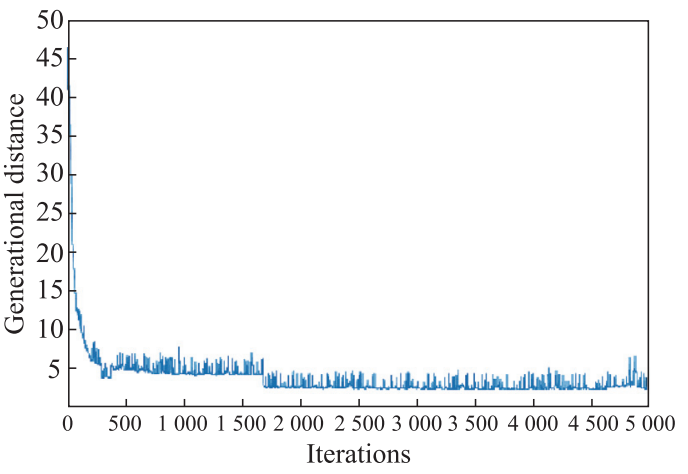

(a) HS algorithm

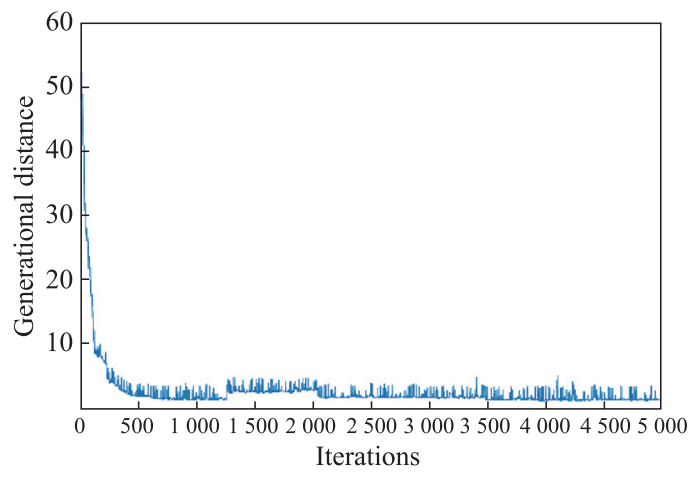

(b) HS+OBL algorithm

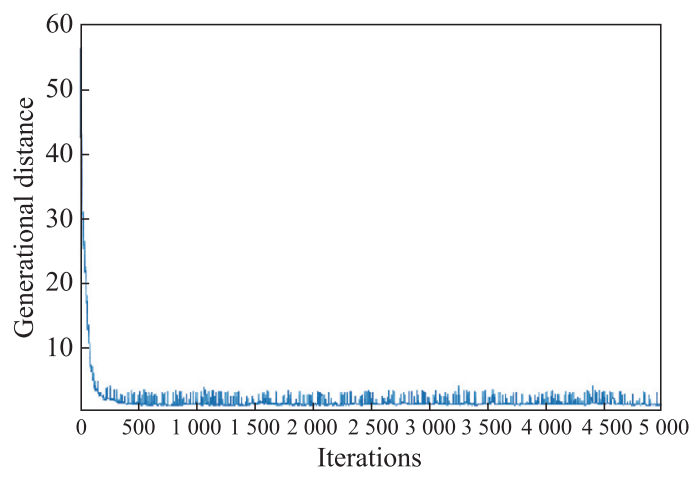

(c) HS+OBL+halving algorithm

Fig. 8 Convergence curves of DTLZ3

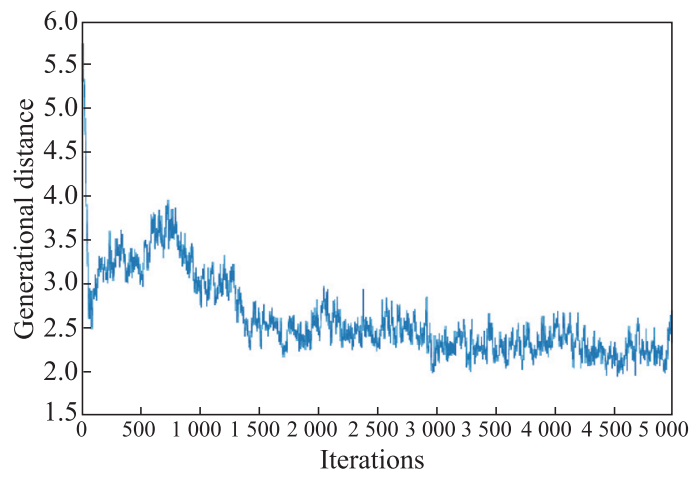

(a) HS algorithm

Fig. 7 Convergence curves of DTLZ2 


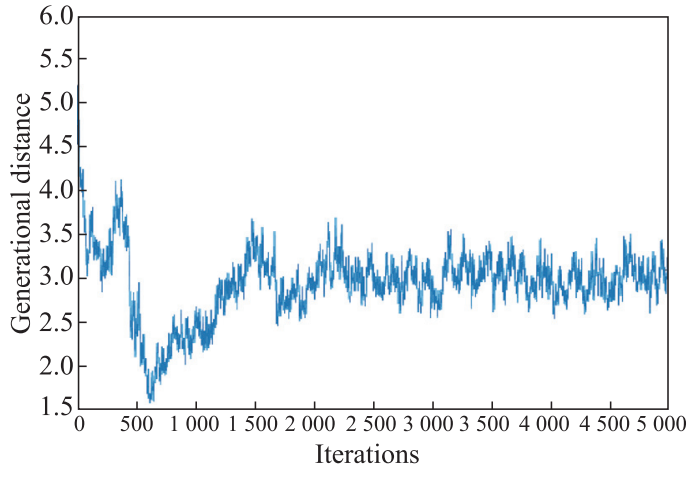

(b) $\mathrm{HS}+\mathrm{OBL}$ algorithm

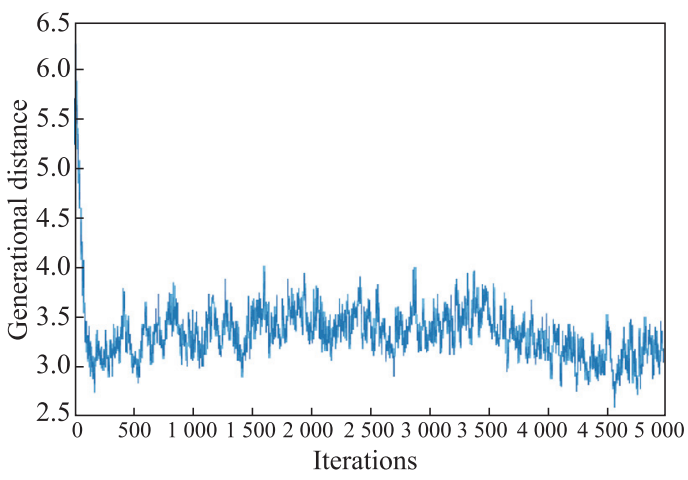

(c) HS+OBL+halving algorithm

Fig. 9 Convergence curves of DTLZ4

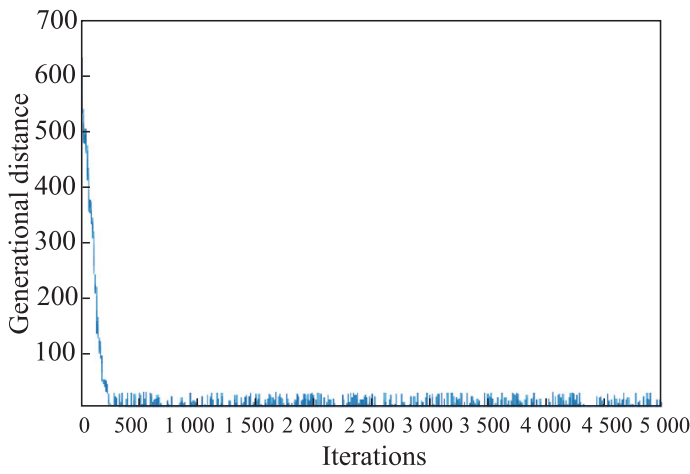

(a) HS algorithm

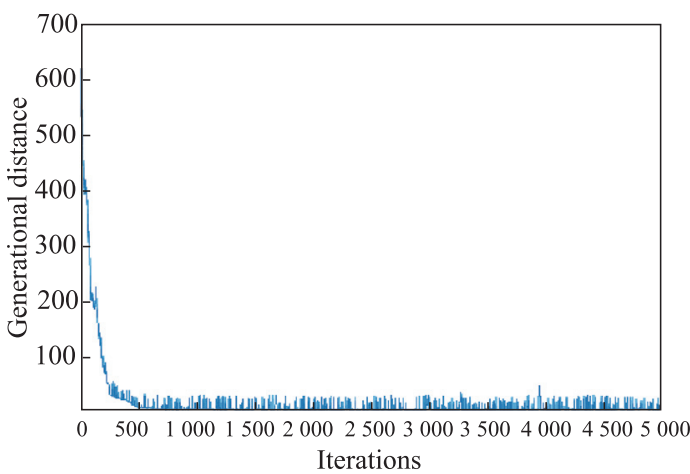

(b) HS+OBL algorithm

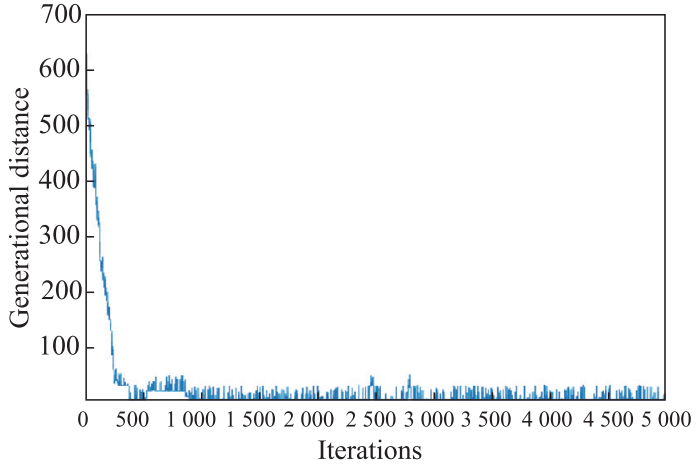

(c) HS+OBL+halving algorithm

Fig. 10 Convergence curves of DTLZ7

According to the IGD metrics, as depicted in Table 4, the best performances are optimized by the OGHS except DTLZ2. Therefore, the OBL and the recursive halving technique embedded in the OGHS not only improve the distribution of solutions but also accelerate the convergence speed during the evolution.

Table 4 IGD mean and variance values at generation 100,500 , and 3000

\begin{tabular}{|c|c|c|c|c|c|}
\hline \multirow{2}{*}{ Problem } & \multirow{2}{*}{ Algorithm } & \multirow{2}{*}{ Value } & \multicolumn{3}{|c|}{ Generation } \\
\hline & & & 100 & 500 & 3000 \\
\hline \multirow{6}{*}{ DTLZ1 } & \multirow{2}{*}{ HS } & Mean & 0.1096 & 0.0604 & 0.0560 \\
\hline & & Variance & 0.0019 & 0.0005 & 0.0015 \\
\hline & \multirow{2}{*}{$\mathrm{HS}+\mathrm{OBL}$} & Mean & 0.0840 & 0.0608 & 0.0477 \\
\hline & & Variance & 0.0011 & 0.0005 & 0.0007 \\
\hline & \multirow{2}{*}{ OGHS } & Mean & 0.0885 & 0.0608 & 0.0347 \\
\hline & & Variance & 0.0010 & 0.0007 & 0.0005 \\
\hline \multirow{6}{*}{ DTLZ2 } & \multirow{2}{*}{ HS } & Mean & 0.1239 & 0.0995 & 0.0913 \\
\hline & & Variance & 0.0071 & 0.0027 & 0.0031 \\
\hline & \multirow{2}{*}{$\mathrm{HS}+\mathrm{OBL}$} & Mean & 0.1114 & 0.0979 & 0.0962 \\
\hline & & Variance & 0.0061 & 0.0030 & 0.0028 \\
\hline & \multirow{2}{*}{ OGHS } & Mean & 0.1043 & 0.0913 & 0.0916 \\
\hline & & Variance & 0.0034 & 0.0025 & 0.0023 \\
\hline \multirow{6}{*}{ DTLZ3 } & \multirow{2}{*}{ HS } & Mean & 0.3665 & 0.2389 & 0.1190 \\
\hline & & Variance & 0.0268 & 0.0084 & 0.0046 \\
\hline & \multirow{2}{*}{$\mathrm{HS}+\mathrm{OBL}$} & Mean & 0.1842 & 0.1484 & 0.1059 \\
\hline & & Variance & 0.0074 & 0.0053 & 0.0042 \\
\hline & \multirow{2}{*}{ OGHS } & Mean & 0.1485 & 0.1033 & 0.0971 \\
\hline & & Variance & 0.0040 & 0.0029 & 0.0029 \\
\hline \multirow{6}{*}{ DTLZ4 } & \multirow{2}{*}{ HS } & Mean & 0.0834 & 0.0535 & 0.0495 \\
\hline & & Variance & 0.0200 & 0.0059 & 0.0054 \\
\hline & \multirow{2}{*}{$\mathrm{HS}+\mathrm{OBL}$} & Mean & 0.0682 & 0.0524 & 0.0484 \\
\hline & & Variance & 0.0093 & 0.0046 & 0.0032 \\
\hline & \multirow{2}{*}{ OGHS } & Mean & 0.0631 & 0.0499 & 0.0401 \\
\hline & & Variance & 0.0100 & 0.0061 & 0.0026 \\
\hline \multirow{6}{*}{ DTLZ7 } & \multirow{2}{*}{ HS } & Mean & 1.4139 & 1.0486 & 0.2493 \\
\hline & & Variance & 0.0910 & 0.1125 & 0.0109 \\
\hline & \multirow{2}{*}{$\mathrm{HS}+\mathrm{OBL}$} & Mean & 0.9009 & 0.6360 & 0.2537 \\
\hline & & Variance & 0.0836 & 0.0636 & 0.0085 \\
\hline & \multirow{2}{*}{ OGHS } & Mean & 0.6620 & 0.5088 & 0.2236 \\
\hline & & Variance & 0.0381 & 0.0332 & 0.0058 \\
\hline
\end{tabular}

\subsection{Benchmark instance tests}

Three representative MOFJSP instances are considered in our experiments, namely two types of benchmarks 
(medium scale, i.e., problem $10 \times 10$, and large scale, i.e., problem $15 \times 10$ ) taken from Kacem, et al. [35,56] and a benchmark (larger scale, i.e., problem $20 \times 10$ ) presented by Brandimarte [8]. To demonstrate the efficiency of our algorithms, the outcomes of the OGHS are compared with those of four well-known algorithms, i.e., PSO+SA [31], the artificial immune algorithm (AIA) [57], the multiobjective genetic algorithm (MOGA) [58] and the Pareto- based discrete artificial bee colony (P-DABC) algorithm [59].

\subsubsection{Computational results}

Comparison results on two Kacem problems with three objectives is detailed in Table 5. Furthermore, a comparison of experiment results on Brandimarte problem $20 \times 10$ is listed in Table 6.

Table 5 Comparison results for Kacem instances

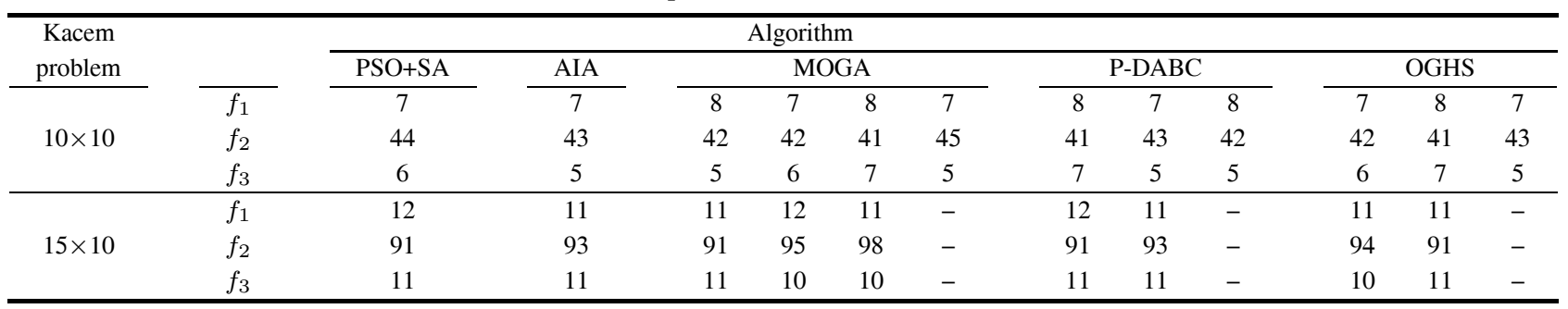

Table 6 Comparison results for Brandimarte instance

\begin{tabular}{|c|c|c|c|c|c|c|c|c|c|c|c|c|c|c|c|c|c|}
\hline \multirow{2}{*}{$\begin{array}{c}\text { Brandimarte } \\
\text { problem }\end{array}$} & & \multirow{2}{*}{ AIA } & \multirow{2}{*}{\multicolumn{10}{|c|}{ P-DABC }} & \multicolumn{5}{|c|}{ OGHS } \\
\hline & & & & & & & & & & & & & 1 & 2 & 3 & 4 & 5 \\
\hline \multirow{3}{*}{$20 \times 10$} & $f_{1}$ & 312 & 311 & 313 & 319 & 324 & 411 & 417 & 424 & 431 & 474 & 484 & 346 & 453 & 463 & 466 & 479 \\
\hline & $f_{2}$ & 2591 & 2288 & 2286 & 2280 & 2279 & 2265 & 2253 & 2240 & 2230 & 2223 & 2210 & 2260 & 2215 & 2214 & 2212 & 2210 \\
\hline & $f_{3}$ & 306 & 299 & 311 & 307 & 307 & 349 & 360 & 386 & 402 & 444 & 454 & 342 & 412 & 422 & 438 & 454 \\
\hline
\end{tabular}

\subsubsection{Analysis of results}

Table 5 shows that the OGHS algorithm obtains the nondominated solutions for the two Kacem instances. For the problem $10 \times 10$, the OGHS algorithm outperforms $\mathrm{PSO}+\mathrm{SA}$. In addition, the results are not worse than $\mathrm{P}$ DABC and MOGA. The solution, i.e., $\left\{f_{1}=7, f_{2}=43\right.$, $\left.f_{3}=5\right\}$, dominates the solution $\left\{f_{1}=7, f_{2}=45, f_{3}=\right.$ $5\}$ generated by MOGA. For the larger-scale problem $15 \times 10$, the OGHS algorithm achieves two non-dominated solutions. For the problem $20 \times 10$, one non-dominated solution set, where the candidate solutions are denoted with 1-5 successively, is shown in Table 6. The five solutions of the OGHS are not dominated by the results of AIA, and the three solutions, i.e., $\left\{f_{1}=346, f_{2}=2260\right.$, $\left.f_{3}=342\right\},\left\{f_{1}=453, f_{2}=2215, f_{3}=412\right\}$ and $\left\{f_{1}=479, f_{2}=2210, f_{3}=454\right\}$, dominate the results $\left\{f_{1}=411, f_{2}=2265, f_{3}=349\right\},\left\{f_{1}=474\right.$, $\left.f_{2}=2223, f_{3}=444\right\}$ and $\left\{f_{1}=484, f_{2}=2210\right.$, $\left.f_{3}=454\right\}$ obtained by P-DABC, respectively. Furthermore, the non-dominated solution which provides the minimal total workload of machines is improved.

In summary, the OGHS algorithm is either outstanding or the same for the three test instances. In addition, it can obtain more than one scheme corresponding to the same non-dominated solution for all the problems in most cases. The proposed algorithm thus performs well in larger-scale problems.

\section{Decision-making of the most appropriate solution}

In engineering practice, choosing a satisfying solution to formulate a scheduling program from an NDSS is a crucial step. The decision-making of the most appropriate solution for the MOFJSP from a finite set of alternatives is an MADM problem. TOPSIS, which includes seven steps [60], is a useful technique in dealing with MADM problems in the real world [61-63]. The detailed procedures of TOPSIS for the $20 \times 10$ problem are explained as follows.

Step 1 The decision matrix $\boldsymbol{D}=\left\{x_{i j}\right\}_{m \times n}$ of the problem $20 \times 10$ is expressed as

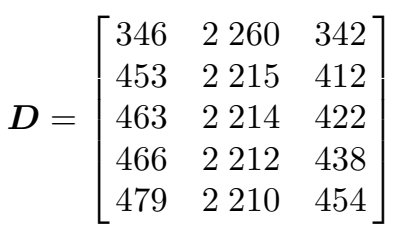

where $x_{i j}$ indicates the performance of the $i$ th alternative with respect to the $j$ th attribute.

Step 2 The normalized value $\boldsymbol{R}=\left\{r_{i j}\right\}_{m \times n}$ can be calculated using the linear normalization formula:

$$
r_{i j}=\frac{x_{i j}}{\sum_{i=1}^{m} x_{i j}} .
$$

Furthermore, the decision matrix $\boldsymbol{R}$ can be expressed in matrix format as 


$$
\boldsymbol{R}=\left[\begin{array}{lll}
0.1568 & 0.2034 & 0.1654 \\
0.2053 & 0.1994 & 0.1992 \\
0.2098 & 0.1993 & 0.2041 \\
0.2111 & 0.1991 & 0.2118 \\
0.2170 & 0.1989 & 0.2195
\end{array}\right]
$$

Step 3 The weighted normalized value $\boldsymbol{V}=\left\{v_{i j}\right\}_{m \times n}$ is calculated as

$$
\boldsymbol{V}=\boldsymbol{R} \cdot \boldsymbol{\omega}^{\mathrm{T}}
$$

where $\boldsymbol{\omega}=\left(\boldsymbol{\omega}_{1}, \boldsymbol{\omega}_{2}, \boldsymbol{\omega}_{3}\right)$, and $\boldsymbol{\omega}_{j}$ is the weight of the $j$ th attribute, $0 \leqslant \boldsymbol{\omega}_{j} \leqslant 1$ and $\sum_{j=1}^{3} \omega_{j}=1$.

Step 3a AHP is adopted to calculate a priori weight $\boldsymbol{\omega}^{a}$, and the a priori attribute weight matrix is listed in Table 7.

Table 7 A priori attribute weight matrix

\begin{tabular}{cccc}
\hline & Makespan & $\begin{array}{c}\text { Total } \\
\text { workload }\end{array}$ & $\begin{array}{c}\text { Critical } \\
\text { machine } \\
\text { workload }\end{array}$ \\
\hline Makespan & 1.0000 & 0.2500 & 0.1667 \\
Total workload & 4.0000 & 1.0000 & 0.3333 \\
Critical machine workload & 6.0000 & 3.0000 & 1.0000 \\
\hline
\end{tabular}

Table 7 shows the approximation value of the maximum eigenvalue $\lambda_{\max } \approx 3.0537$, the consistency index $\mathrm{CI}=$ 0.0268 and the random consistency index $\mathrm{RI}=0.58$. Accordingly, the consistency ratio is

$$
\mathrm{CR}=\frac{\mathrm{CI}}{\mathrm{RI}}=0.0462<0.1
$$

Hence, the normalized a priori weight value is

$$
\boldsymbol{\omega}^{a}=(0.0852,0.2706,0.6442) .
$$

Step 3b The objective weight of attribute $\boldsymbol{\omega}^{c}$ is calculated by using the information entropy of the system, $E_{j}$, where the mathematical expressions are provided as follows:

$$
\begin{gathered}
E_{j}=-\frac{1}{\ln n} \sum_{i=1}^{m} r_{i j} \ln r_{i j} \\
\omega_{j}^{c}=\frac{\left(1-E_{j}\right)}{\sum_{j=1}^{n}\left(1-E_{j}\right)} .
\end{gathered}
$$

The result is $\boldsymbol{E}=(1.4592,1.4650,1.4609)$. The objective weight is $\boldsymbol{\omega}^{c}=(0.3315,0.3357,0.3328)$.

Step 3c With integration of the subjective and objective weights, the weight distribution values of each attribute are received from the following formula:

$$
\omega_{j}=\frac{\omega_{j}^{a} \omega_{j}^{c}}{\sum_{j=1}^{n} \omega_{j}^{a} \omega_{j}^{c}} .
$$

Thus, the weight is $\boldsymbol{\omega}=(0.0847,0.2724,0.6429)$.

Consequently, the decision matrix is

$$
\boldsymbol{V}=\left[\begin{array}{lll}
0.0133 & 0.0554 & 0.1063 \\
0.0174 & 0.0543 & 0.1281 \\
0.0178 & 0.0543 & 0.1312 \\
0.0179 & 0.0542 & 0.1362 \\
0.0184 & 0.0542 & 0.1411
\end{array}\right]
$$

Step 4 The values for the ideal solution, $\boldsymbol{V}^{+}$, and the nonideal solution, $\boldsymbol{V}^{-}$, defined respectively as $\boldsymbol{V}^{+}=$ $\min \left\{v_{i j} \mid i=1,2,3,4,5\right\}$, and $\boldsymbol{V}^{-}=\max \left\{v_{i j} \mid i=\right.$ $1,2,3,4,5\}$, are obtained:

$$
\begin{gathered}
\boldsymbol{V}^{+}=(0.0133,0.0542,0.1063) \\
\boldsymbol{V}^{-}=(0.0184,0.0554,0.1411) .
\end{gathered}
$$

Step 5 The Euclidean distances $\overline{\boldsymbol{A}}^{+}$and $\overline{\boldsymbol{A}}^{-}$from $\boldsymbol{V}^{+}$ and $\boldsymbol{V}^{-}$, respectively, are calculated by using the following equations for alternative $i(i=1,2,3,4,5)$ :

$$
\begin{aligned}
& \bar{A}_{i}^{+}=\sqrt{\sum_{j=1}^{n}\left(v_{i j}-V_{j}^{+}\right)^{2}} \\
& \bar{A}_{i}^{-}=\sqrt{\sum_{j=1}^{n}\left(v_{i j}-V_{j}^{-}\right)^{2} .}
\end{aligned}
$$

As a result,

$$
\overline{\boldsymbol{A}}^{+}=\left(\begin{array}{lllll}
0.0012 & 0.0222 & 0.0253 & 0.0303 & 0.0352
\end{array}\right)
$$

and

$$
\overline{\boldsymbol{A}}^{-}=\left(\begin{array}{lllll}
0.0352 & 0.0131 & 0.0100 & 0.0051 & 0.0012
\end{array}\right) .
$$

Step 6 The relative proximity $C_{i}^{*}$ to the ideal solution is calculated as follows:

$$
C_{i}^{*}=\frac{\bar{A}_{i}^{-}}{\bar{A}_{i}^{+}+\bar{A}_{i}^{-}}
$$

where $0 \leqslant C^{*} \leqslant 1$. Therefore, the relative proximity is $C^{*}=(0.9670,0.3711,0.2833,0.1441,0.0330)$.

Step 7 The best alternative is ranked according to $C_{i}^{*}$ in descending order. The priority ranking for candidate solutions calculated by using the TOPSIS is as follows:

$$
1 \prec 2 \prec 3 \prec 4 \prec 5 \text {. }
$$

Therefore, the 1st candidate solution is considered as the most appropriate solution. In addition, the most appropriate scheduling scheme is described in Table 8. In the table, 
as for the form $M k\left(t_{i, j}, t f_{i, j}\right), M k$ denotes the processing machine, $t_{i, j}$ denotes the start time, and $t f_{i, j}$ denotes the completion time. The symbol " -" indicates that the process does not exist. This approach enables the construction of the most appropriate scheduling scheme for the MOFJSP according to the preferences of the decisionmaker. Compared with the above, with respect to the AIA algorithm, the most appropriate solution is a nondominated one. Meanwhile, with respect to the P-DABC, the selected candidate solution dominates one result.

Table 8 Most appropriate scheduling scheme for problem $20 \times 10$ presented by Brandimarte $\left(f_{1}=346, f_{2}=2260, f_{3}=342\right)$

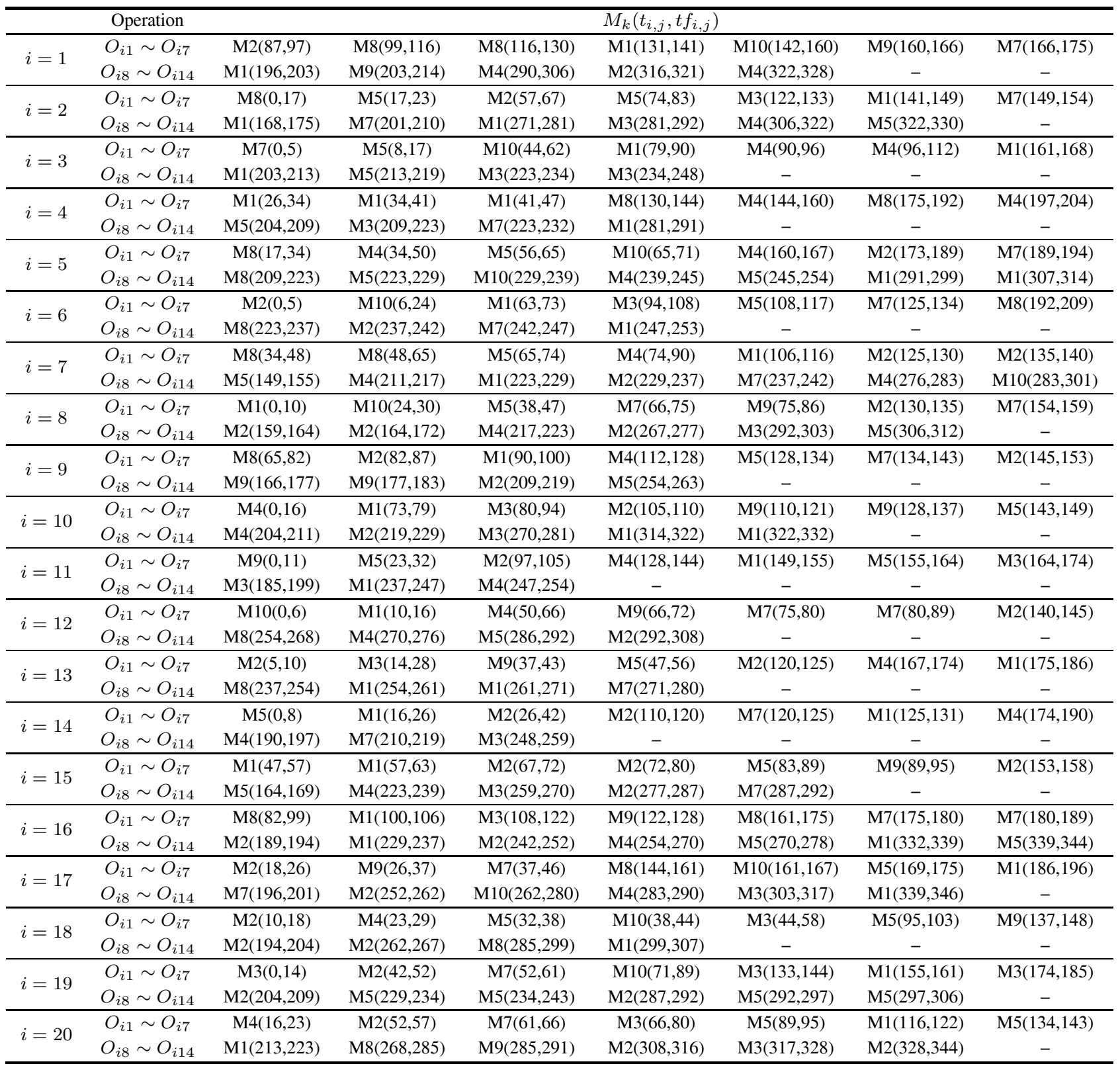

\section{Conclusions and future work}

(i) The OGHS algorithm, which integrates HS and OBL, is proposed for solving the MOFJSP. The theoretical contribution is reflected in the improved performance of the proposed algorithm. Meanwhile, the practical contribution is that the complicated MOFJSPs are successfully solved by using the proposed algorithm. (ii) In the OGHS algorithm, Pareto optimality is employed to solve the non-dominated solution set for the MOFJSP. OBL is developed to enhance the performance of the algorithm through improving the distribution quality of the individuals in the initial population. Furthermore, recursive halving based on OBL is utilized to shrink the neighbourhood space and to accelerate convergence.

(iii) A practical implication for the MOFJSP is that 
TOPSIS is adapted to decide the most appropriate scheduling scheme.

Further examinations need to be conducted in consideration of the following aspects.

(i) The robustness and the time efficiency of the proposed algorithm should be further improved.

(ii) Some excellent heuristics and hybrid algorithms are developed to solve the MOFJSP, especially for large-scale instances. Importantly, the performance of different coding schemes and algorithm strategies directly affects the efficiency and effectiveness of the algorithm. Further work should investigate thoroughly the relevant performance measures corresponding to different strategies.

(iii) A simultaneous investigation of additional objectives and uncertainties for the MOFJSP is vital.

\section{References}

[1] ZUO Y, GU H Y, XI Y G. Modified bottleneck-based heuristic for large-scale job-shop scheduling problems with a single bottleneck. Journal of Systems Engineering and Electronics, 2007, 18(3): 556-565.

[2] LI K, YANG S L. Heuristic algorithms for scheduling on uniform parallel machines with heads and tails. Journal of Systems Engineering and Electronics, 2011, 22(3): 462-467.

[3] SAIDI M M, FATTAHI P. Flexible job shop scheduling with tabu search algorithms. International Journal of Advanced Manufacturing Technology, 2007, 32(5-6): $563-570$.

[4] SHEN L, DAUZÈRE-PÉRÈS S, NEUFELD J S. Solving the flexible job shop scheduling problem with sequence-dependent setup times. European Journal of Operational Research, 2018, 265(2): $503-516$.

[5] LIU Q, CHEKEM F O, ZHAN M, et al. A hybrid fruit fly algorithm for solving flexible job-shop scheduling to reduce manufacturing carbon footprint. Journal of Cleaner Production, 2017, 168: 668-678.

[6] GAREY M R, JOHNSON D S, SETHI R. The complexity of flow-shop and job-shop scheduling. Mathematics of Operations Research, 1976, 1(2): 117-129.

[7] BRUCKER P, SCHLIE R. Job-shop scheduling with multipurpose machines. Computing, 1990, 45(4): 369-375.

[8] BRANDIMARTE P. Routing and scheduling in a flexible jobshop by taboo search. Annals of Operations Research, 1993, 41(3): $157-183$.

[9] SEYED H A R, ZANDIEH M. A new biogeography-based optimization (BBO) algorithm for the flexible job shop scheduling problem. International Journal of Advanced Manufacturing Technology, 2012, 58(9-12): 1115-1129.

[10] ISHIKAWA S,KUBOTA R, HORIO K. Effective hierarchical optimization by a hierarchical multi-space competitive genetic algorithm for the flexible job-shop scheduling problem. Expert Systems with Applications, 2015, 42(24): 9434 -9440.

[11] YUAN Y, XU H, YANG J D. A hybrid harmony search algorithm for the flexible job shop scheduling problem. Applied Soft Computing, 2013, 13(7): 3259-3272.

[12] SINGH M R, MAHAPATRA S S. A quantum behaved particle swarm optimization for flexible job shop scheduling. Computers \& Industrial Engineering, 2016, 93: 36-44.

[13] MATI Y, REZG N, XIE X L. An integrated greedy heuristic for a flexible job shop scheduling problem. Proc. of the IEEE International Conference on Systems, Man, and Cybernetics, 2001: $2534-2539$.

[14] LIU A J, MICHELE P, JOHN F. Scheduling optimization of task allocation in integrated manufacturing system based on task decomposition. Journal of Systems Engineering and Electronics, 2016, 27(2): 422-433.

[15] NOUIRI M, BEKRAR A, JEMAI A, et al. An effective and distributed partical swarm optimization algorithm for flexible job-shop scheduling problem. Journal of Intelligent Manufacturing, 2018, 29(3): 603-615.

[16] SHEN X N, HAN Y, FU J Z. Robustness measures and robust scheduling for multi-objective stochastic flexible job shop scheduling problems. Soft Computing, 2017, 21(21): $6531-$ 6554.

[17] ZHANG Y, WANG J, LIU Y. Game theory based real-time multi-objective flexible job shop scheduling considering environmental impact. Journal of Cleaner Production, 2017, 167(20): $665-679$.

[18] ZITZLER E, THIELE L. Multi-objective evolutionary: a comparative case study and the strength Pareto approach. IEEE Trans. on Evolutionary Computation, 1999, 3(4): 257-271.

[19] CORNE D W, JERRAM N R, KNOWLES J D, et al. PESAII: region-based selection in evolutionary multi-objective optimization. Proc. of the 3rd Annual Conference on Genetic and Evolutionary Computation, 2001: 283-290.

[20] SRINIVAS N, DED K. Multi-objective optimization using non-dominated sorting genetic algorithm. Evolutionary Computation, 1994, 2(3): $221-248$.

[21] DEB K, PRATAP A, AGARWAL S, et al. A fast and elitist multi-objective genetic algorithms: NSGA-II. IEEE Trans. on Evolutionary Computation, 2002, 6(2): $182-197$.

[22] LI J Q, PAN Q K, XIE S X. A hybrid variable neighborhood search algorithm for solving multi-objective flexible job shop problems. Computer Science and Information Systems, 2010, 7(4): $907-930$.

[23] RAHMATI S H A, ZANDIEH M, YAZDANI M. Developing two multi-objective evolutionary algorithms for the multiobjective flexible job shop scheduling problem. International Journal of Advanced Manufacturing Technology, 2013, 64(58): $915-932$.

[24] WANG X J, LI W F, ZHANG Y. An improved multi-objective genetic algorithm for fuzzy flexible job-shop scheduling problem. International Journal of Computer Applications in Technology, 2013, 47(2-3): 280-288.

[25] ROHANINEJAD M, KHEIRKHAH A. A hybrid multiobjective genetic algorithm based on the ELECTRE method for a capacitated flexible job shop scheduling problem. International Journal of Advanced Manufacturing Technology, 2015, 77(1-4): $51-66$.

[26] JIA S, HU Z. Path-relinking tabu search for the multi-objective flexible job shop scheduling problem. Computers \& Operations Research, 2014, 47(9): $11-26$.

[27] ZHANG G H, SHAO X Y, LI G, et al. An effective hybrid particle swarm optimization algorithm for multi-objective flexible job-shop scheduling problem. Computers \& Industrial Engineering, 2009, 56(4): $1309-1318$.

[28] VILCOT G, BILLAUT J C. A tabu search algorithm for solving a multicriteria flexible job shop scheduling problem. International Journal of Production Research, 2011, 49(23): $6963-$ 6980.

[29] LI J Q, PAN Q K, SUGANTHAN P N, et al. A hybrid tabu 
search algorithm with an efficient neighborhood structure for the flexible job shop scheduling problem. International Journal of Advanced Manufacturing Technology, 2011, 52(5-8): 683-697.

[30] KAPLANOGLU V. An object-oriented approach for multiobjective flexible job-shop scheduling problem. Expert System with Applications, 2016, 45(1): 71-84.

[31] XIA W J, WU Z M. An effective hybrid optimization approach for multi-objective flexible job-shop scheduling problems. Computers \& Industrial Engineering, 2005, 48(2): 409425.

[32] LOW C YY, YIP Y, WU T H. Modeling and heuristics of FMS scheduling with multiple objectives. Computers \& Operations Research, 2006, 33(3): 674-694.

[33] GAO K Z, SUGANTHAN P N, PAN Q K, et al. Paretobased grouping discrete harmony search algorithm for multiobjective flexible job shop scheduling. Information Sciences, 2014, 289(24): 76-90.

[34] TAN K C, CHIAMS S C, MAMUN A A, et al. Balancing exploration and exploitation with adaptive variation for evolutionary multi-objective optimization. European Journal of Operational Research, 2009, 197(2): 701-713.

[35] KACEM I, HAMMADI S, BORNE P. Approach by localization and multi-objective evolutionary optimization for flexible job-shop scheduling problems. IEEE Trans. on Systems, Man and Cybernetics, 2002, 32(1): 1-13.

[36] ALTANNAR C, PANOS M, PARDALOS. A survey of recent developments in multi-objective optimization. Annals of Operations Research, 2007, 154(1): 29-50.

[37] BAUMGARTNER U, MAGELE C, RENHART W. Pareto optimality and particle swarm optimization. IEEE Trans. on Magnetics, 2004, 40(2): $1172-1175$.

[38] PARETO V. Manual of political economy. SCHWIER A S Trans. New York: Anguitus M. Kelly, 1971.

[39] KONAK A, COIT D W, SMITH A E. Multi-objective optimization using genetic algorithms: a tutoria. Reliability Engineering and System Safety, 2006, 91(9): $992-1007$.

[40] GEEM Z W, KIM J H, LOGANATHAN G V. A new heuristic optimization algorithm: harmony search. Simulation, 2001, 76(2): $60-68$.

[41] LEE K S, GEEM Z W. A new meta-heuristic algorithm for continuous engineering optimization: harmony search theory and practice. Computer Methods in Applied Methanics and Engineering, 2005, 194(36-38): 3902-3933.

[42] MAHDAVI M, FESANGHARY M, DAMANGIR E. An improved harmony search algorithm for solving optimization problems. Applied Mathematics and Computation, 2007, 188(2): $1567-1579$.

[43] MAHAMED G H O, MEHRDAD M. Global-best harmony search. Applied Mathematics and Computation, 2008, 198(2): $643-656$

[44] TIZHOOSH H R. Opposition-based learning: a new scheme for machine intelligence. Proc. of the International Conference on Computational Intelligence for Modeling, Control and Automation, and International Conference on Intelligent Agents, Web Technologies and Internet Commerce, 2005, 1: $695-$ 701.

[45] WANG H, LIU Y, ZENG S Y, et al. Opposition-based particle swarm algorithm with Cauchy mutation. Proc. of the IEEE Congress on Evolutionary Computation, 2007: 4750-4756.

[46] SHAHZAD F, BAIG A R, MASOOD S, et al. Oppositionbased particle swarm optimization with velocity clamping.
Advances in computational intelligence. Berlin, Heidelberg: Springer, 2009, 61: 339-348.

[47] RAHNAMAYAN S, TIZHOOSH H R, SALAM M. Opposition-based differential evolution. IEEE Trans. on Evolutionary Computation, 2008, 12(1): $64-79$.

[48] ERGEZER M, SIMON D, DU D. Oppositional biogeographybased optimization. Proc. of the IEEE International Conference on Systems, Man and Cybernetics, 2009: 1009-1014.

[49] ERGEZER M, SIMON D. Oppositional biogeography-based optimization for combinatorial problems. Proc. of the IEEE Congress on Evolutionary Computation, 2011: 1496-1503.

[50] GEN M, TSUJIMURA Y, KUBOTA E. Solving job-shop scheduling problem using genetic algorithms. Proc. of the 16th International Conference on Computer and Industrial Engineering, 1994: 576-579.

[51] BEAN J C. Genetic algorithms and random keys for sequencing and optimization. ORSA Journal on Computing, 1994, 6(2): $154-160$.

[52] OUYANG H B, GAO L Q, ZOU D X, et al. Exploration ability study of harmony search algorithm and its modification. Control Theory \& Applications, 2014, 31(1): 57-65.

[53] TAN K C, YANG Y J, GOH C K. A distributed cooperative coevolutionary algorithm for multi-objective optimization. IEEE Trans. on Evolutionary Computation, 2006, 10(5): 527 - 549.

[54] DEB K, THIELE L, LAUMANNS M, et al. Scalable multiobjective optimization test problems. Proc. of the Congress on Evolutionary Computation, 2002: 825-830.

[55] LI H, ZHANG Q F. Multi-objective optimization problems with complicated Pareto sets, MOEA/D and NSGA-II. IEEE Trans. on Evolutionary Computation, 2009, 13(2): 284-302.

[56] KACEM I, HAMMADI S, BORNE P. Pareto-optimality approach for flexible job-shop scheduling problems: hybridization of evolutionary algorithms and fuzzy logic. Mathematics and Computers in Simulation, 2002, 60(3-5): 245-276.

[57] BAGHERI A, ZANDIEH M, MAHDAVI I, et al. An artificial immune algorithm for the flexible job-shop scheduling problem. Future Generation Computer Systems, 2010, 26(4): 533 541.

[58] WANG X J, GAO L, ZHANG C Y, et al. A multi-objective genetic algorithm based on immune and entropy principle for flexible job-shop scheduling problem. International Journal of Advanced Manufacturing Technology, 2010, 51(5-8): $757-$ 767.

[59] LI J Q, PAN Q K, GAO K Z. Pareto-based discrete artificial bee colony algorithm for multi-objective flexible job shop scheduling problems. International Journal of Advanced Manufacturing Technology, 2011, 55(9-12): 1159-1169.

[60] OPRICOVIC S, TZENG G H. Compromise solution by MCDM methods: a comparative analysis of VIKOR and TOPSIS. European Journal of Operational Research, 2004, 156(2): $445-455$.

[61] HWANG C L, YOON K. Multiple attribute decision making. Berlin: Springer-Verlag, 1981.

[62] SHEN F, MA X, LI Z, et al. An extended intuitionistic fuzzy TOPSIS method based on a new distance measure with an application to credit risk evaluation. Information Sciences, 2018, 428: 105 - 119 .

[63] SILVIA C, ANTONELLA C, JOAQUÍN I, et al. K-out-of-n systems: an exact formula for the stationary availability and multi-objective configuration design based on mathematical programming and TOPSIS. Journal of Computational and Applied Mathematics, 2018, 330(1): $1007-1015$. 


\section{Biographies}

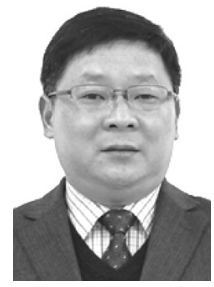

PENG Jiangang was born in 1970. He is currently a Ph.D. and an associate research fellow at School of Mechanical Engineering in Hefei University of Technology, China. His main research interests include production planning and scheduling, quality function deployment, decision-making analysis, fuzzy theory and multi-objective optimization algorithm.

E-mail: peng_jiangang@163.com

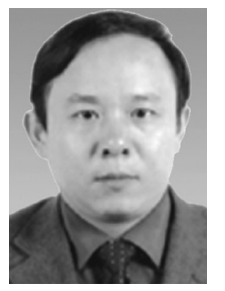

LIU Mingzhou was born in 1968. He is currently a professor and a Ph.D. candidate supervisor in manufacturing process monitoring and control at School of Mechanical Engineering in Hefei University of Technology, China. His main research interests include theory, analysis \& decision method of industrial engineering system, monitoring and control of manufacturing process, modeling and simulation of manufacturing system, modern integrated manufacturing system, facilities planning and logistics engineering and computer integrated manufacturing system.

E-mail: LiuMingZhou0551@163.com

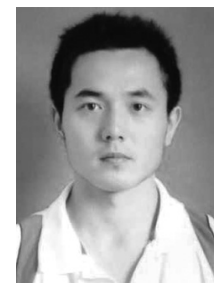

ZHANG Xi was born in 1985. He received his Ph.D. degree at School of Mechanical Engineering in 2015, Hefei University of Technology, China. His main research interests include production scheduling and intelligent manufacturing, uncertainty and multi-objective optimization.

E-mail: isaachft@126.com

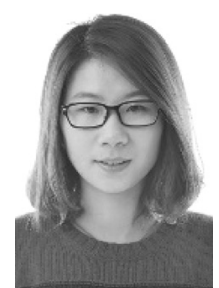

LING Lin was born in 1987. She received her $\mathrm{Ph} . \mathrm{D}$. degree from Hefei University of Technology in 2014. She works in Hefei University of Technology from 2014. Her research interests include material flow control and intelligent manufacturing system.

E-mail: linglin8787@123.com 